\title{
Gouldian Arguments \& the Sources of Contingency
}

\author{
Alison McConwell \& Adrian Currie \\ Penultimate Version, forthcoming in Biology \& Philosophy
}

\begin{abstract}
'Gouldian arguments' appeal to the contingency of a scientific domain to establish that domain's autonomy from some body of theory. For instance, pointing to evolutionary contingency, Stephen Jay Gould suggested that natural selection alone is insufficient to explain life on the macroevolutionary scale. In analysing contingency, philosophers have provided source-independent accounts, understanding how events and processes structure history without attending to the nature of those events and processes. But Gouldian Arguments require source-dependent notions of contingency. An account of contingency is source-dependent when it is indexed to (1) some pattern (i.e, microevolution or macroevolution) and (2) some process (i.e., Natural Selection, species sorting, etc.). Positions like Gould's do not turn on the mere fact of life's contingency - that life's shape could have been different due to its sensitivity to initial conditions, path-dependence or stochasticity. Rather, Gouldian arguments require that the contingency is due to particular kinds of processes: in this case, those which microevolutionary theory cannot account for. This perspective clarifies both debates about the nature and importance of contingency, and empirical routes for testing Gould's thesis.
\end{abstract}

\section{Introduction}

It is often claimed that history is contingent, and that this matters for the natures of historical explanation, science and evidence. Philosophers approach contingency with a focus on the outcomes of contingent processes: some outcome or process is contingent when it instantiates a particular modal profile, understood abstractly. Its modal properties are structured in the right way: it could be stochastic, or sensitive to initial conditions, for instance. However, some circumstances call for a different approach, one which fills out the details leading to said outputs. This requires specification of the causes of contingency, that is, the particular kinds of processes to blame. Some outcome or process has the modal properties it has because of the causal networks it is embedded in; a selection process could be contingent due to mutational ordering, for instance. Let's call the first approach —one 
which requires only that an output have a particular modal profile, restricting the relevant processes by their capacity to structure outputs in the relevant way-source-independent. Let's call the second — which demands that the processes be specified in more detail—-sourcedependent.

In this paper, we'll establish the importance of source-dependent accounts of contingency by focusing on an argument of Stephan Jay Gould's, which denies the sufficiency of microevolutionary theory in accounting for macroevolutionary pattern (or, if you'd prefer, microevolutionary processes to produce macroevolutionary pattern). We'll highlight the inadequacy of source-independence and show how a source-dependent perspective clarifies philosophical debate, Gould's argument, and its empirical support.

Gould argued that understanding microevolutionary process is insufficient for understanding macroevolutionary pattern. Natural selection occurs at a local ecological level, heritable traits which endow advantages in survival or reproduction spread throughout populations. However, Gould doubted such microlevel processes could be responsible for biology's 'big picture': speciation, radiations, mass extinctions, and so forth. His argument appealed to the contingency of these patterns and processes. However, the mere (that is, source-independent) contingency of evolution cannot establish Gould's thesis. Rather, his position requires that macroevolution be contingent due to processes or events which are outside of microevolutionary theory. This requires a source-dependent conception of contingency.

We'll proceed as follows. In section 2 we'll summarize philosophical accounts of contingency and distinguish between source-dependence and source-independence. In section 3 we argue that because natural selection is itself a source of contingency, merely establishing source-independent contingency is insufficient for Gould's arguments. In section 
4, we show how the distinction between source-dependence and source-independence clarifies a difference between Turner (2011a) and Beatty (2006)'s discussion of Gould. Finally, in section 5, we discuss how source-dependence sheds light on attempts to test Gould's claim. Specifically, we argue that whether instances of biological convergence support or undermine Gouldian positions depends upon which processes are responsible for those convergences.

Before delving in, it will be useful to contrast our focus with others. First, we should distinguish between several prominent Gouldian themes. Gould is frequently concerned with denying that life has a goal $(1989,233)$. He is critical of the idea that Homo sapiens' evolution was inevitable; that we are somehow special or (god forbid) divinely ordained. In other contexts, Gould worries about the emphasis of evolution by natural selection in a range of domains - not simply macroevolution $(2002,505,541 \mathrm{ff})$. We focus only on his argument that the theory of evolution by natural selection doesn't have the goods to explain macroevolutionary patterns (2002, 579; also see Beatty 2006).

A second point of departure concerns the relationship between contingency and explanation. Philosophers often examine the nature of historical explanation by asking, for example, why evolutionary and other historical explanations take a 'narrative' form, or emphasizing the fragility of historical events (see Beatty 2011, Currie 2014, Roth 2008, Sterelny forthcoming). In such contexts, we investigate the connection between how history unfolds and what explanatory forms are applicable to it. According to Beatty, for instance, the dependence of evolutionary outcomes on prior events, and our inability to predict such outcomes, is appropriately captured by narrative explanation. In contrast, we are interested in which theoretical resources are applicable to which empirical domains. 
Third, philosophers appeal to contingency to underwrite notions of historical kinds (Ereshefsky 2014, Griffiths 1999, Smart 1959, Hull 1976). On such views, kind-membership is not determined by intrinsic properties, but rather by partaking in a shared history-Bailey is a member of Felis catus not because he and other F. catus share catty properties, but because they bear the right historical relationships to one another: they form chains of inheritance. Such views and ours have different targets: we are interested in understanding a kind of epistemological argument which we take Gould to make, whereas these philosophers focus on the ontological question of historical kinds.

So, how might attention to source-dependence establish Gould's argument? We'd better first understand the distinction between source-dependence and source-independence.

\section{Source-Independence and Dependence}

Consider two broad strategies for understanding the relationship between an event or outcome and its antecedent causes. On the one hand, we might conceive of the causes minimally: the event has some modal properties (it is necessary, contingent, path-dependent, etc...) and the causes are such that the event has those properties. This is source-independent. Outcomes are grouped by modal profile. On the other hand, we might explain how the causes actually structure the outcome: we provide detail of the antecedent process leading to the outcome. This is a source-dependent conception: we group outcomes in terms of the kinds of processes which produce them.

'Contingency' is a modal property of some event, pattern or process. At base, contingent patterns or outcomes could have been different (Ben-Menahem 1997) - the causal webs in which they are imbedded could have influenced and structured them in different ways. The distinction between source-independent and dependent contingency turns on how we 
conceive of those causal webs, of the processes to blame for the outcomes we're interested in. A conception is source-independent when we require the outcome to be modally structured in a certain way; a source-dependent conception demands that this modal structure is due to a particular kind of process_-what in particular is to blame for the outcome in question's modal profile? In this section, we'll clarify the distinction and briefly survey philosophicallargely source-independent — accounts of contingency.

Evolutionary outcomes are contingent (if they are) in virtue of being the results of contingent processes. Source-dependence and independence are two ways of conceiving of contingency, which turn on how we describe those processes. An account of contingency is source-independent when we require only that the processes in question structure outcomes in a particular way. For instance, we can describe an outcome as path-dependent-it turns on event ordering — without specifying which events, or explaining what the dependence is between those events and the outcome. Alternatively, an account of contingency is sourcedependent when the process itself possesses certain features. Here we might say that an evolutionary outcome is contingent due to mutational ordering, that is, the emergence of a particular genotype requires a particular sequence of mutations. In the former sourceindependent case, all that is required for an outcome to be contingent is for it to have certain modal properties, e.g. the outcome could-have-been-different. In the latter source-dependent case, we specify the physical processes and events in virtue of which the outcome has those modal properties. For instance, at a large scale, life's unfolding could have been different because its path depends upon the timing and nature of mass-extinctions, and these are themselves sensitive to events like extra-terrestrial impacts, changes in climate, and so on.

And so, accounts of contingency specify (1) an outcome's modal profile and (2) the processes which are to blame for that profile. Source dependence and independence come 
apart in terms of the second aspect. Source-independent contingency involves specifying the processes minimally; all that is required is that they are sufficient for producing the modal profile in question. Source-dependent conceptions provide more detail about sources - the contingency-producing-processes — by, for instance, identifying the physical processes in question.

An outcome's being contingent source dependently or independently, then, is a matter of how we describe or conceive of it; it is an explanatory, not metaphysical or ontological, distinction. And so, we shouldn't ask whether a particular event or process is contingent in source-dependent or independent terms, but instead whether it is better, given the relevant context, to conceive of it source-dependently or independently. Moreover, the distinction between source-dependence and independence is graded. Some accounts of contingency (see, for instance, Powell \& Mariscal 2015) require that macroevolution could have been different because of low probability events. Here, a minimal requirement is placed on the sources of contingency: not only must they structure outcomes in the relevant manner, but they must be of low probability. There may very well be interesting border-line cases, but we'll treat the distinction as discrete for simplicity’s sake ${ }^{1}$.

Philosophers have focused on source-independent notions of contingency, but for an important set of Gould's arguments, we must conceive of contingency source-dependently.

\footnotetext{
1 'Stochastic processes' are possible examples. A system is stochastic when its evolution involves (or is best modeled using) at least one random variable. As a referee points out, some arguments for macroevolutionary autonomy — particularly those using the MBL model — explicitly contrasted stochastic processes with those ruled by Natural Selection. The basic strategy was to show that a random-undirectedprocess could produce the same patterns in the fossil record as those which are usually blamed on Natural Selection. One might wonder whether stochasticity should be classified as a source-dependent or independent notion of contingency. These pre-Wonderful Life arguments seem to classify stochastic processes due to the patterns they cause; and what is required to count as the relevant process is that they be undirected. This strikes us as minimally source-dependent. If this is true, then there is evidence of Gould thinking in a source-dependent way. This would, in effect, help our case that source-dependence is needed to follow through Gouldian arguments as we discuss in section 3 .
} 
Before characterizing Gouldian arguments, we should make good on our claim that philosophers have conceived of contingency source-independently.

Philosophical analysis of contingency often (and perhaps problematically) centres on a thought-experiment Gould introduced in Wonderful Life. Here, life is measured out across a length of tape, before being rewound and replayed (1989). Beatty (2006) notes two variations on the thought experiment, and develops a notion of contingency tracking each.

On the first of Gould's replays, we vary things somewhat: tweaking the initial conditions, or perhaps restarting from a different stage — we replay a similar tape (Gould WL 1989a, 227 and 289). We often imagine this source-dependently. Shift the meteor which exterminated the dinosaurs slightly to the left. As the tape unfolds, the meteor misses, the KPg extinction doesn't occur, thus neither do the mammalian radiation nor the evolution of Homo sapiens. However, Beatty and others analyse the notion abstractly, sourceindependently: on this kind of replay, all that is required for contingency is that changes in initial conditions lead to changes downstream.

Beatty calls this 'causal-dependence': to say that an evolutionary outcome is contingent is to say that the outcome necessarily depends on some prior states (Beatty 2006, 339). The existence of later states is, in some fashion, sensitive to earlier states; variations in starting conditions and changes in the ordering of events constrain future outcomes:

Alter any earlier event... and evolution cascades into a radically different channel (Gould 1989a, 51).

Causal dependence has been explored more-or-less abstractly (Desjardins 2011, Inkpen \& Turner 2012). The focus is source-independent: it largely does not matter what is responsible for the pattern—we needn't specify a particular process—so long as changes upstream make the relevant differences downstream. 
On the second kind of replay, we hold everything fixed, that is, we replay the same tape (1989a, 278). On this version, unpredictability, the existence of prior states does not guarantee later states. Here's Gould:

But the diversity of possible itineraries does demonstrate that the eventual results cannot be predicted at the outset. Each step proceeds for a cause, but no finale can be specified from the start and none would ever occur a second time in the same way, because any pathway proceeds through thousands of improbable stages. (WL 1989a, $51)$.

Identical starting points lead to different outcomes on replays, rendering the prediction of a particular outcome extremely difficult if not impossible. ${ }^{2}$ Unpredictability is tricky. An outcome can be unpredictable due to a lack of knowledge on our part. But this is not the notion philosophers have in mind. Turner complains: "unpredictability is infelicitous [as a term] because that depends on the epistemic limitations of humans" (2011a, 67, also Millstein 2000). ${ }^{3}$ Alternatively, unpredictability could be due to a failure of determinism. That is, the complete state of the world at one time (plus the laws of nature) could be insufficient to determine the state of the world at some other time. However, this reading should be also eschewed because "the unpredictability version of contingency...is not tantamount to any mysterious sort of indeterminism. It does not deny that evolutionary outcomes can be explained" (Beatty 2006, 345). Finally, a pattern could be unpredictable given some set of processes (Powell 2009). Macroevolutionary patterns, speciation say, might not be predictable given facts relevant to the process of Natural Selection - the environment, the

\footnotetext{
${ }^{2}$ A referee worried about our attribution of both replay experiments to Gould, claiming that Gould's position is better captured by the second replay (unpredictability). We are not primarily interested in Gouldinterpretation here, instead we're focused on how contingency has been conceived of in light of his work. Although an interesting endeavor, establishing whether Gould really thought causal dependency was contingency is not the aim of this paper. In this section we are merely showing how contingency is construed in a source-independent way.

${ }^{3}$ Indeed, Turner recommends switching to 'causal insufficiency'. We don't find the term as problematic as Turner does, but we are understanding it in his terms.
} 
mix of traits within populations, and so forth. This final option, it seems to us, is potentially source-dependent, rather than source-independent. On a source-independent reading, something is unpredictable if any particular set of processes are insufficient for its occurrence, while a source-dependent reading will specify the processes in question.

In summary, philosophical analysis of contingency has emphasized sourceindependence, conceptions where contingency holds just in case the relevant outcomes are influenced and structured in the right way. Source-independent accounts do not specify a process, but rather its effect: the source is effectively black-boxed. Such accounts have undoubtedly shed light on biological possibility but, as we shall see, they are limited when it comes to 'Gouldian Arguments', to which we now turn.

\section{Gouldian Arguments \& Contingency}

Gould claimed that shape of life's contingency undermines the view that natural selection can explain macro-evolutionary patterns. In this section, we'll argue that a sourceindependent characterisation of contingency is insufficient for such an argument.

An important facet of Gould and his collaborators' objections to the idea that macroevolution can be understood in microevolutionary terms appeals to the contingency of evolution (see Gould 1989a, 2002). However, the structure of Gould's argument (or perhaps arguments) and what he intended the meaning of 'contingency' to be, are both ambiguous and have proven fertile ground for philosophical reflection, development and disagreement (see Beatty 1982, 1995, 2006, Desjardins 2011a, 2011b, Turner 2011a, 2011b, 2015). We want to know how appeals to contingency support the kind of claims Gould made about macroevolution. 
Gould often attempted to reign in what he saw as the overreach of evolutionary theory emphasizing natural selection. This took several forms: from his critique of adaptationism (Gould and Lewontin 1979, Forber 2009), to his anti-gradualist views on speciation (Eldredge \& Gould 1972), to his emphasis on the role of development and constraint (Gould 1977, 1980a, 1989b, 2002). And finally, to our focus: evolutionary history's contingency. Gould's opponent maintains that extinction (mass or otherwise), radiations, speciation and other aspects of what GG Simpson called the 'tempo \& mode' of evolution, can be accounted for in the main by appeal to evolution by natural selection. As Kim Sterelny has put it, “... the view that the evolution of species and species lineages is just an aggregate of local ecological processes of the kind we have observed" 2001, 81; also see Grantham 2007). Just as a population's immediate environment will shuffle and shape the proportion of traits in that population, these same processes are to blame for lineages' 'life histories'. On such a view, many traits will at some point be present in populations, and evolution is such that the fittest traits will go to fixation ${ }^{4}$.

Gouldian arguments are often best understood in non-binary terms, that is, they make relative significance claims about the causal forces in question. Beatty (1997) links evolutionary contingency to relative significance debates in biology. Biologists seek a correct (rather than the correct) account of a domain. They then try to establish their account's scope. This explains why Gould was perfectly happy with what he referred to as the 'first phase' of the Modern Synthesis $(2002,519)$, which involved the active combination of different theories (i.e. Mendelian genetics and Darwinian evolution). However, Gould was deeply unhappy with the second phase of the synthesis, wherein a confidence in microevolutionary theory led to the systematic trivialization and marginalization of

\footnotetext{
${ }^{4}$ For discussion of various types of adaptationism, see Godfrey-Smith (2001), Lewens (2009).
} 
macroevolution (580). Aggressively pushing the explanatory reach of natural selection is not in the spirit of the theoretical pluralism implied by relative significance debates.

In this vein, Gould's critique was not intended to show that the microevolutionary theory of natural selection was wrong per se, but rather to keep it in check. If macroevolution is contingent, then natural selection is insufficient to explain life's shape — but what does contingency have to do with Natural Selection's explanatory reach? The connection between evolutionary contingency and selection's insufficiency needs to be drawn out.

We will use a simple, informal account of the machinery of evolutionary theory to argue that establishing source-independent contingency is insufficient for Gouldian Arguments. The same argument could be cashed out more formally, and more carefully — using population genetics, say—but by our lights informality will suffice.

In brief, natural selection can itself be a source of contingency: evolutionary outcomes are sensitive to initial conditions (such as the heredity of traits) and are path dependent (such as the requirement that trait evolution and environments match). ${ }^{5}$ Moreover, if the facts relevant to selection are black-boxed, evolutionary outcomes are unpredictable. Thus, both of Beatty's notions of contingency can be generated by natural selection.

The bare bones of evolution via natural selection — at least according to the 'recipe' view (Lewontin 1970, Godfrey-Smith 2009) —includes three ingredients. ${ }^{6}$ First, a population must vary—-members must have different traits. Second, that variation must make a difference to survival and reproductive success among members. Third, individuals must breed true, that

\footnotetext{
${ }^{5}$ While natural selection can be a source of contingency, we discuss stochastic processes, such as mutation, drift, and species sorting as possible sources of contingency in section 4 .

${ }^{6}$ This is a purposefully simplistic treatment of a complex topic. See, for instance, Brandon (1990), Lewontin (1970) and (Okasha 2006) for differing accounts of the 'recipe'. There are more formal ways of understanding Natural Selection which do not overtly rely on recipes: see Bourrat (2014) for example. We assume that the points we make here are transferable mutatis mutandis.
} 
is, there must be a connection between an individual possessing a trait, and its offspring possessing it. If these conditions hold, namely, variation, differential fitness, and heritability, traits which have a positive influence on the survival and reproductive success of individuals will spread throughout the population over subsequent generations.

Consider primate colour vision. Unlike most mammals, many old-world monkeys and all apes are trichromatic, that is, have relatively rich, three-colour vision. ${ }^{7}$ What explains this? One hypothesis points to our ancestors' frugivorous habits (Osorio et al 1996, Regan et al 2001, De Araujo et al 2006). In a fruit-eating environment, traits which increase one's chances of identifying fruit-ripe fruit especially — provide a selective advantage. Possessing a richer chromatic template would allow individual primates to distinguish between fruit and the foliage around them, as well as between more or less ripe fruit. If heritable trichromatic vision were to arise in a population of frugivores (and assuming this didn't bring other fitness costs), then turning natural selection's crank would be sufficient to produce a population with trichromatic vision.

Merely showing that evolutionary outcomes are contingent does not in itself undermine the applicability of microevolutionary theory to macroevolution, because natural selection's ingredients can themselves be sources of contingency. Let us explain.

Take causal dependence. Following Desjardins (2011a), events are contingent in terms of causal dependence when they are sensitive to initial conditions, or path-dependent, or both. Natural selection, even in our basic rendition, can handle causal dependence so long as the dependency's sources fall within its remit. For example, according to our adaptationist explanation of trichromacy, the trait's evolution was contingent upon its heritability. Had it

\footnotetext{
${ }^{7}$ This simplifies somewhat, as new-world monkeys are often polymorphic (Osario et al 2004, Regan et al 2001). It is still unclear whether trichromancy evolved independently in the two lineages.
} 
not been heritable - if, for instance, a parent's trichromacy gave no reason to expect the same in offspring - then the trait would not have spread. A trait's evolution by natural selection can also exhibit path-dependency. For instance, for trichromacy to be an adaptation to fruitforaging, the evolution and spread of trichromatic vision must occur after inhabiting the relevant frugivorous environment. If our ancestors possessed three-pigment vision before targeting fruit, then the trait did not spread through the population in response to that environment (it could be, perhaps, an exaptation rather than an adaptation). The importance of timing and order in adaptationist explanation shows that path-dependence is built right into its structure. Moreover, if factors like fitness and heritability are omitted—if we don't know which traits are likely to be stable across generations - then evolution's path becomes unpredictable.

The process of natural selection, then, can be a source of contingency. The theory implies that evolutionary outcomes are sensitive to initial conditions, such as a trait's heritability, that such outcomes are path-dependent, such as matching the emergence of an adaptation to an environment, and that we must factor in fitness and heritability to predict evolutionary outcomes. And so, the mere fact of an event's being contingent has no necessary connection to natural selection failing to account for it. More is needed. Establishing mere (sourceindependent) contingency cannot undermine the applicability of some body of theory (i.e. Natural Selection) to a domain. It must also be shown that the contingent patterns and outcomes in question cannot be accounted for using that theory.

Much of the foregoing has focused on source-independent accounts of contingency. Recall that an account of contingency is source-independent just when some system's exhibiting that contingency is purely a matter of how its evolutionary trajectory or modal profile is structured. Alternatively, an account is source-dependent when we specify the 
process(es) responsible for contingency. It is our contention that philosophers have conflated these notions with negative results. In the next section, we show how failure to distinguish between source-dependence and independence has led to confusion. We aim to clarify a complex difference in how Beatty (2006) and Turner (2011a) understand contingency.

\section{Beatty and Turner on Contingency}

Turner (2011a) and Beatty (2006) include different evolutionary forces under the banner of 'contingency'. Beatty considers two microevolutionary processes - drift and mutation - taking Gould to categorize the latter as contingent and the former as not. While Beatty wonders whether the exclusion of drift in favor of mutation is artificial $(2006,345$, 360), his focus mostly remains at the microevolutionary scale. Turner disagrees with Beatty's grain of analysis, and instead argues that the macroevolutionary process of species sorting is where the action is. As we'll show, why Beatty, Gould and Turner might include some and exclude other processes as being contingent is clarified once recast sourcedependently. We'll first briefly characterize the potential sources of contingency at play.

First, mutation matters because it is an important source of the variation selection works with (Jacob 1977, Carlson 2011). While most geneticists view 'mutation' as a technical term referring to changes in individual genes (see Muller 1922), generally mutational effects include qualitative or discontinuous shifts in expression (Carlson 2011,3). These changes are often not advantageous, that is, they are not biased by environmental conditions. Second, drift occurs when changes in gene frequency are due to factors that affect the persistence of allelic variation in populations but are not sensitive to environmental fit. This yields a ratio of some allele in a population that is not due to its "adaptive superiority" and is more-or-less 
independent of natural selection (Okasha 2015) ${ }^{8}$. Third, species sorting, as Turner (2011a) puts it, is a macroevolutionary analogue of random drift—certain species persist (or cease to persist) in a non-directed fashion.

Whereas mutation and drift are microevolutionary processes that affect the proportion of traits across generations within a lineage, species sorting is a macroevolutionary process occurring among groups of lineages. Unbiased sorting results in the persistence of some species over others, and the sorting process is not due to suitability for environmental conditions (for contrast see ‘biased species sorting' in Langenheder and Székley 2011). Mutation, drift, and species sorting are all process which interfere with evolution by Natural Selection. Beatty and Turner appear to differ on which of these processes are contingent. Should we ask who is right? As we'll argue, a source-dependent perspective resolves the issue.

On the one hand, Beatty includes mutation as a source of contingency, but excludes drift. He includes mutation because the generation of particular mutations constrain the range and direction of variation. He cites their random occurrence as the reason why evolution is unpredictable (2006, 347). (Beatty is to some extent engaging in Gouldian exegesis, thus he excludes drift is because he thinks Gould excludes it, though he wonders whether the exclusion of drift in favour of mutation is artificial $(2006,345,360))$.

On the other hand, Turner distinguishes between effects at the macro and micro levels (2011a, 69). He excludes microevolutionary processes, such as mutation and drift, as sources of contingency; the contingency of evolutionary history requires something of macroevolutionary processes: "Evolutionary contingency is the random or unbiased sorting

\footnotetext{
${ }^{8}$ The relationship between selection and drift, and the nature of the latter, is a vexed topic which we avoid here (see Plutynski 2007).
} 
of entire lineages." (ibid). For Turner, historical processes are contingent when they are both stochastic and unconstrained. $^{9}$

Under source-independent definitions of contingency, the differences between Turner and Beatty's accounts seem odd. They puzzle over how to characterize evolution according to the patterns generated by non-adaptive processes at the micro and macro levels. The result is an ambiguity. It is unclear whether they disagree over the level-micro or macroevolutionat which contingency is more significant, or whether they disagree over a particular evolutionary process's ability to generate evolutionary contingency.

Appealing to source-dependency clarifies Turner and Beatty's differences. Recall that an account of contingency is source-dependent when it is indexed to [1] a particular pattern (that is, the thing which is contingent), and [2] a particular process (that is, the thing which generates the contingency). Beatty and Turner target whether evolutionary contingency follows the sorts of patterns generated by random mutation, or drift, or species sorting. These processes generate particular patterns of evolutionary change. However, to exclude a process from being a source of contingency, we need to specify which pattern we care about, and then empirically demonstrate that the excluded process does not have a (relatively substantial) effect on that pattern. For example, if Turner cares about the macroevolutionary pattern generated by unbiased species sorting, then if he wants to exclude microevolutionary

\footnotetext{
${ }^{9}$ The latter notion - unconstrained - is required because in principle stochasticity can yield inevitable outcomes, if there are constraints in place. For example, consider a case where at some maximum number of lineages, those lineages would be randomly selected for extinction. Although the ordering of extinctions may be unbiased in that case, it will always result in no existing lineages at all. Perhaps Turner adds 'unconstrained' because he is worried about cases of evolutionary convergence (and associated notions of inevitability) that are often considered cases against Gould's project. However, we argue in Section 5 that a source-dependent view of contingency demonstrates that some convergences are due to the generative capacity of developmental systems. This works in Gould's favour. That is, the sources of convergences and divergences matter.
} 
processes (e.g. mutation and drift), he must show that those processes have little effect on that pattern. ${ }^{10}$

And so, from a source-dependent perspective, there are two possible versions of the difference between their views. First, it might be reasonable to think that Beatty and Turner care about different patterns. That is, perhaps Beatty cares about microevolutionary scales. If so, he is concerned whether selection is insufficient for evolutionary change at that level by appealing to processes such as mutation. And, Turner might care about macroevolutionary patterns. That is, to show that selection is insufficient to capture the shape of life's history by appeal to the significance of unbiased species sorting at that level. However, if Beatty and Turner care about different patterns at different scales, then they are talking past one another. If so, this would not be a disagreement per se, but rather a difference in grain of analysis.

Second, if Beatty and Turner are concerned with the same pattern, then their disagreement is empirical; it is a matter of whether the putative sources they name are in fact to blame for the production of the relevant pattern. Consider their assessments of the evolutionary experiment by Travisano et al. (1995). This experiment aims to test Gould's ideas about the role of contingency in evolution, exploring the effects of chance, history, and adaptation in evolution $(1995,88)$. By testing populations of $E$. coli, Travisano et al. attempt to determine the relative contributions of those influences. Beatty complains that they include random drift in addition to mutation under the category of chance $(2006,353)$, whereas Turner worries that they exclude unbiased sorting at the macrolevel $(2011 \mathrm{a}, 75) .{ }^{11}$ The relevancy of these concerns depend upon Travisano et al.'s aim. If we are concerned with microevolutionary pattern, and the insufficiency of selection to account for it, then

\footnotetext{
${ }^{10}$ In correspondence, Turner claims to be inclusionary about sources of contingency at the macro and micro scales.

${ }^{11}$ Turner does note that if contingency is macrolevel stochasticity, then there is no way a microevolutionary experiment can show how much contingency is in evolution $(2011 \mathrm{a}, 75)$.
} 
mutation (and drift) do the job just fine-Turner's worry about species-sorting misses the mark.

However, if we are concerned with macroevolutionary pattern, and the insufficiency of selection to account for it, then there is a disagreement. Beatty must show that mutation has effects significant to life's shape on the macroevolutionary scale. ${ }^{12}$ However, from a sourcedependent perspective, he (or Gould) has no obvious grounds to exclude drift from his analysis. This is because thinking about the sources of contingency emphasizes commonalities between mutation and drift, e.g. that they both generate patterns which natural selection, or other biased processes, cannot account for. And so, source-dependency also explains why Travisano et al. lump together mutation and drift- they were concerned with measuring the significance of patterns one might contrast with patterns of adaptive fit. In other words, since both drift and mutation generate patterns not biased to the environment, that commonality justifies measuring their influence in combination against the significance of selection. However, Travisano et al. claim to establish the influence of chance concerning particular E. coli traits, such as the evolution of cell size $(1995,89)$. That conclusion does not directly support the claim that mutation affects macroevolutionary pattern. Although at times they discuss the influence of chance on particular lineages (88), it is difficult to establish which scale - micro or macro-Travisano et al. are primarily concerned with.

As for Turner, if he were to think that unbiased species sorting is alone responsible for evolutionary contingency at the macroscale, then he would have to show that mutation and drift do not significantly affect macroevolutionary patterns of change. If it is the case that

\footnotetext{
12 Notably, Beatty does discuss the Losos group macroevolution study insofar as he thinks it relates to the causal dependence sense of contingency $(2006,353)$. He sees the Travisano experiment as a microevolutionary study (ibid). Since Beatty identifies mutation as a source of the historical or causal dependence sense of contingency in footnote 12 (p.347) perhaps, then, there is disagreement with Turner concerning the sources of contingency at the macrolevel.
} 
Travisano et al. are trying to discuss macroevolutionary pattern, then Turner's complaint is compelling. Travisano et al. claim that the bacterial populations show "parallel and even convergent evolution," which is traditionally considered a mark against Gould's project $(1995,89)$. However, if they had found a way to include the effects of unbiased species sorting, perhaps their results would have been different. That is, perhaps the significance of chance effects would have increased. Overall, establishing the influences of particular processes on macroevolutionary patterns is a difficult task. An experiment such as Travisano et al.'s is one way to probe these hypotheses, but the timescale required to capture patterns of macroevolution renders the results indirectly relevant at best. ${ }^{13}$

Finally, here's a third option. Beatty and Turner might be pluralists; they're simply articulating different source-independent notions of contingency. If so, their positions are of limited relevant to Gould's argument: for as we've seen, such arguments require sourcedependent conceptions of contingency.

In summary, to think about contingency source-dependently is to identify a causal process (mutation, drift, species sorting) and a pattern (micro, macro), and discuss the sufficiency or otherwise of that process in generating that pattern. Up to this point, it looks as though the sources of contingency will be whatever causes a pattern of evolutionary change that natural selection, or other biased sorting processes for that matter, cannot account for on some evolutionary scale. What follows from this is that mutation, drift and unbiased species sorting are all potential sources of evolutionary contingency. ${ }^{14}$ The significance of

\footnotetext{
${ }^{13}$ Even Travisano et al. worry that experiments can only span over short stretches of time, which suggests they aim to test macroevolution $(1995,89)$.

${ }^{14}$ Gould generally expressed concern over the role of randomness because of its association with lack of pattern, order, and control ( $W L 1989,51)$. But the fact that stochastic processes introduce random variables does not mean that evolution is unintelligible as events in the past must be appropriately related to events that come later. Turner makes a good case for the stochasticity of processes in evolution as neutral on the traditional (in)determinism debate $(2011,72-3)$.
} 
evolutionary contingency sourced by each of these processes will depend upon which pattern of change one is concerned with. The interesting question, it seems, is not whether one source or another counts as contingent, but whether those sources are in fact to blame for the patterns that concern us. How to answer that question is the topic of the next section.

\section{The Primate, the Quokka, \& the Cuttlefish}

We've seen how a source-dependent perspective matters for understanding Gouldian Arguments and the nature of contingency. Moreover, the distinction between source dependence and independence clarifies otherwise confusing differences in philosophical accounts. In this section we demonstrate that source-dependence matters for testing Gould's claim that patterns of macro-evolution are driven by processes beyond natural selection's reach. How might one gain empirical traction on this thesis? Diverse investigative approaches have been linked to macroevolutionary contingency: models of macroevolutionary processes (Huss 2009), lab-based ‘bottle experiments' (Travisano 1995, Beatty 2006, Desjardins 2011b) and so-called 'natural experiments' (Bromham 2016, Powell \& Mariscal 2014, 2015, Beatty 2006). We're going to focus on an example of the latter and show how the sources of convergence and divergence matter for establishing Gould's thesis.

Biological convergence is a common phenomenon. It involves the evolution of similar (typically adaptive) traits in two more-or-less independent evolutionary histories. As we've discussed, humans and some other primates are trichromats: their visual world is coloured with three pigments. Trichromacy is also present in another group of mammals - the marsupials. This is a homoplasy: a trait which is not present in a common ancestor has evolved in two subsequent descendent lineages.

Proto-mammals were quadchromats, like fish, reptiles and birds today. It is thought that two pigments and their respective cones were lost as early Mesozoic mammals adapted to a 
nocturnal habitat, leaving dichromatic basal mammals. In some of our primate ancestors, presumably in response to shifting into a better-lit (and fruit-rich!) environment, trichromacy evolved. Meanwhile, on another branch of the mammalian tree, some marsupials added another pigment to their visual arsenal (Arrese et al 2002, Arrese et al. 2005, Ebeling, et al. 2010). Interestingly, trichromacy and dichromancy are both present in the group: quokka, quenda, honey-tailed possum and fat-tailed dunnarts are trichromes, but some wallabys (Ebeling et al. 2010) and possums (Vlahos et al. 2014) are not.

Two lineages of mammals separately evolving trichomacy should tell us something about the evolution of that trait specifically, and perhaps about the shape of life overall. Indeed, some prominent authors-Simon Conway-Morris (2003) and George McGhee (2011) in particular-have appealed to widespread convergence in arguing that Gould is wrong about the shape of life. That is, convergence should lead us to think that replaying the tape of life would not lead to different outcomes. It has been pointed out (Sterelny 2005, Currie 2012, Powell \& Mariscal 2014, 2015) that such arguments are far too quick. Such arguments 'lump' (as Powell \& Mariscal put it) convergences together, treating as the same cases which have different sources. Source-dependency makes this point abundantly clear.

Let's assume that Conway-Morris and McGhee are right insofar as life's shape involves frequent convergence. If we read Gould's claim source-independently, then the ubiquity of convergence might indeed be problematic. It might be tempting to think that his view would predict wide-spread divergence in biological traits.

However, this would be a mistake. What matters for Gouldian arguments are the sources of contingency and thus, on the flip-side, the sources of convergence. That is, we should ask whether the processes which are to blame for frequent convergence are those captured by microevolutionary theory that emphasizes selection. We'll keep our focus on trichromacy. 
To begin, it's important to notice that convergences are sensitive to description (Hall 2012, Currie 2012, 2013, Powell \& Mariscal 2015, Inkpen \& Turner 2012). We can consider trichromacy in very coarse terms: a visual system using three pigments. With finer-grained descriptions, we include which specific pigments. Interestingly, there is reason to think that (for at least the dunnarts) marsupials use a different pigment-range to us (Ebeling et al. 2010). Moreover, we might include the niche in which the trait evolved - did primates and quokka evolve trichromancy due to frugivorous environments? Apparently not: quokka eat grass, shrubs and leaves, and there is no indication of their having frugivorous ancestors. ${ }^{15}$ Quoting Ebeling et al:

... a similarly plausible [to primates] 'feeding hypothesis' does not exist for marsupials. There is no obvious reason why wallabies should be diachromatic, but the related quokka trichromatic, a species with very similar habitat and lifestyle...(2010, e14231)

In other contexts, we might worry about the resources involved in the inheritance and development of the trait. These different levels are in principle (and often are actually) decoupled (Hall 2012, Currie 2014). For instance, to some extent quokka and primates utilize the same developmental resources for trichromacy, ${ }^{16}$ but quokka didn't evolve their colorsense for frugivorous environments. Is it, then, a convergence? On some levels (development, number of pigments) yes, on others (niche, specific pigments) no.

Now, does this single case of convergence count for or against Gould's thesis? This doesn't turn on whether trichromacy's presence in both Quokka and primates is a

\footnotetext{
${ }^{15}$ Arrese et al (2002) suggest that trichromancy in marsupials is basal - that is, it was retained in the marsupial line and not the placental. This seems unlikely, given the relative rarity of trichromancy across marsupials and the novel developmental route in that lineages (see footnote 10).

${ }^{16}$ To some extent: where primates trichromacy utilized an ancestral gene (RH2) for generating their third pigment, dunnarts (and presumably other marsupial trichromats) seem not to. Ebeling et al. (2010) suggests that the RH1 gene, which in other lineages is expressed on in cone development, could have been copied and coopted in marsupials. And so, the developmental convergence is not highly fine-grained, however primates and marsupials still utilize the same developmental network for trichromacy.
} 
convergence, but on the cause of the convergence: its source. We suspect this case supports Gould. It strikes us as significant that (1) similar developmental resources are utilized in the two lineages and that (2) trichromacy evolved in response to different environmental pressures. Plausibly, each event could be explained separately in microevolutionary terms trichromacy arises in a population, and due to its heritability and fitness spreads. However, this does not capture what is convergent about the two cases. Part of the explanation, surely, is the continuity in developmental resources. The use of similar developmental resources suggests that this option is 'open' to mammalian critters. That is, trichromacy is evolvable in the lineage (Brown 2013). After all, the environments which determined the trait's fitness in the two cases were different, while the developmental resources were the same. The explanation, then, appeals to the generative capacity of the developmental systems, not only natural selection. Vis-à-vis the presence of trichromacy in mammals, we have preliminary evidence that the shape of life depends not only on natural selection, but on the internal developmental resources which happen to have been maintained over time

We think the clincher comes from a case of evolutionary divergence. Much has been made of the convergent similarities between cephalopod and vertebrate eyes. Like us, their visual apparatus are camera-like, sporting an iris, lens, and so forth. And this despite the common ancestor living hundreds of million years before the evolution of the trait in the two lineages (Fernald 2006). However, the visual worlds of cephalopods appear to be colourless (Mäthger et al. 2006) ${ }^{17}$. And this despite their mastery of camouflage and (in the case of cuttlefish) complex light-based signalling. Speculatively, were colour vision available to such a visually focused critter, it would be selected for.

\footnotetext{
${ }^{17}$ There is one recording exception to this: O. aegina. However this is a strange case, where colour vision (which emphasized part of the blue spectrum) appears to be decoupled from the rest of the visual system. If anything, it reinforces the difficulty of evolving colour vision in a cephalopod system.
} 
In short, what matters for quokka and primates vis-à-vis eye colour is not their niche, but their common history which bequeathed them similar developmental resources. The dissimilarity between cuttlefish and mammals is plausibly due to a difference in their histories: cephalopods lack whatever it is you need to evolve color vision (let alone trichromacy). This picture seems to support the claim that the sources of contingency (and convergence) are not microevolutionary. This also demonstrates why attending to the difference between parallelisms and convergence matters. When natural selection explains convergences we see a possible mark against Gould's view of life. Moreover, when developmental constraint plays the role, we see a mark for Gould's view. Daniel Dennett's defence of adaptationism involved dismissing non-adaptive forces in evolution as mere 'frozen accidents' (1995). In explaining the convergence of trichromacy in quokka and primates, and the divergence between them and cuttlefish, we see that such frozen accidents matter. The non-adaptive history of the three lineages plays a necessary role in explaining the evolutionary pattern of colour vision - even though the convergent traits are plausibly adaptations. In this one instance, it seems we should side with Gould.

It is worth re-iterating that debates about macroevolution are debates about the relative significance of various forces and events in shaping life. As such, we should not push for extremes. Moreover, we would need to consider the representativeness of trichromacy if we were to draw any broader conclusions from our discussion; something more systematic would be required to reach a firmer conclusion (something along the lines suggested by Powell \& Mariscal (2015), see also Louis (2016) strike us as a good start). And indeed this says nothing about how source-dependent understandings of contingency might play out in other attempts to test Gould's thesis.

\section{Conclusion}


In discussing contingency, philosophers have followed a divide-and-conquer strategy. By distinguishing between different notions of contingency—causal dependence and unpredictability for instance-different routes for analysis have been opened up. Some (Desjardins, for instance) analyse causal dependence, while others (Turner and Beatty) differ about which forces generate unpredictability. We take ourselves to have shown that this strategy is not illuminative of Gouldian Arguments. There are, however, divisions which are more fruitful in this regard. Namely, identify a causal process and a pattern, and discuss the sufficiency or otherwise of that process in generating that pattern. In other words, consider contingency source-dependently.

We can generalize Gouldian Arguments as follows:

(1) Contingency claim: some natural domain D exhibits contingency,

(2) Connecting Premise: If D is contingent, then some body of theory T is insufficient to explain D,

(3) Therefore, $T$ is insufficient to explain D.

On our view, the structuralist, source-independent accounts of contingency developed by Beatty and others have done much to clarify the first premise of such arguments - they have told us what it means to be contingent and moreover how contingency shapes history. However, by conflating source-dependency and independency the importance of the second premise, establishing that contingency ensures the insufficiency of the relevant theory, has been obscured. To defend the connecting premise we need to show that the kind of contingency the domain exhibits is the sort which the theory in question cannot handle. That is, we must attend to contingency's sources. As we've also seen, this switch in focus clarifies what is going on in attempts to empirically test such arguments. We have here focused on what evolutionary convergence has to teach us about the reducibility of macro to micro- 
evolution. This is only one species of Gouldian Argument (even of those made by Gould!) and only one of the empirically relevant streams. Regardless, what we have seen thus far has the potential to justify Gould's position— - to account for life's tempo and mode our microevolutionary toolkit must be supplemented with macroevolutionary theory.

\section{References}

Arrese, C. A., Hart, N. S., Thomas, N., Beazley, L. D., \& Shand, J. (2002). Trichromacy in Australian marsupials. Current Biology, 12(8), 657-660.

Arrese, C. A., Oddy, A. Y., Runham, P. B., Hart, N. S., Shand, J., Hunt, D. M., \& Beazley, L. D. (2005). Cone topography and spectral sensitivity in two potentially trichromatic marsupials, the quokka (Setonix brachyurus) and quenda (Isoodon obesulus). Proceedings of the Royal Society of London B: Biological Sciences, 272(1565), 791-796.

Beatty, J. (2016). What are narratives good for?. Studies in History and Philosophy of Science Part C: Studies in History and Philosophy of Biological and Biomedical Sciences

---. (2006). Replaying Life's Tape. In The Journal of Philosophy, 103 (7):336-62

---. (1997). Why do biologists argue like they do? In Philosophy of Science, 64. Proceedings of the 1996 Biennial Meetings of the Philosophy of Science Association. Part II: Symposia Papers (1997). S432-S443.

---. (1995). The Evolutionary Contingency Thesis. In Concepts, Theories and Rationality in the Biological Sciences: The Second Pittsburgh-Konstanz Colloquium in the Philosophy of Science, University of Pittsburgh, Oct 1-4, 1993. G. Wolters and J.G. Lennox (Eds.) in collab. With P. McLaughlin. Pittsburgh, PA: University of Pittsburgh Press.

---. (1982). What's Wrong with the Received View of Evolutionary Biology? In P.D. Asquith and R.N. Giere (eds.), PSA 1980, Proceedings of the 1980 Biennial Meetings of the Philosophy of Science Association, Vol. 2. East Lansing, Michigan: Philosophy of Science Association.

Ben-Menahem, Y. (1997). Historical contingency. Ratio, 10(2), 99-107.

Bourrat, P. (2015). How to Read 'Heritability' in the Recipe Approach to Natural Selection. The British Journal for the Philosophy of Science, 66(4), 883-903.

Brandon, R. N. (1990). Adaptation and environment. Princeton University Press.

Bromham, L. (2016). Testing hypotheses in macroevolution. Studies in History and Philosophy of Science Part A, 55, 47-59. 
Brown, R. L. (2013). What evolvability really is. The British Journal for the Philosophy of Science, axt014.

Carlson, Elof Axel (2011). Mutation: The History of an Idea from Darwin to Genomics. Cold Spring Harbour, NY: Cold Spring Harbour University Press.

Conway Morris, Simon (2003). Life's Solution: Inevitable Humans in a Lonely Universe. Cambridge, UK: Cambridge University Press.

Currie, A (under review). Rock, Bone \& Ruin.

Currie, A. (2014a) Narratives, mechanisms and progress in historical science. Synthese 191, no. 6 (2014): 1163-1183.

Currie, A. M. (2014b). Venomous dinosaurs and rear-fanged snakes: homology and homoplasy characterized. Erkenntnis, 79(3), 701-727.

Currie, A. (2012). Convergence, contingency \& morphospace. Biology \& Philosophy, 27(4), 583-593.

Currie, A. (2013). Convergence as evidence. The British Journal for the Philosophy of Science, 64(4), 763-786.

Dennett, D. C. (1995). Darwin's dangerous idea. The Sciences, 35(3), 34-40.

Desjardin, E (2011a). Reflections on Path Dependence and Irreversibility: Lessons from Evolutionary Biology. In Philosophy of Science, 78 (5): 724-38.

Desjardin, E. (2011b). Historicity and experimental evolution. In Biol. Philos. 26: 33964.Ebeling, W., Natoli, R. C., \& Hemmi, J. M. (2010). Diversity of color vision: not all Australian marsupials are trichromatic. PLoS One, 5(12), e14231.

Ereshefsky, M. (2014). Species, historicity, and path dependency. Philosophy of Science, 81(5), 714-726.

Fernald, Russell D. (29 September 2006). "Casting a genetic light on the evolution of eyes". Science 313 (5795): 1914-1918

Forber, P. (2009). Spandrels and a pervasive problem of evidence. Biology \& Philosophy, 24(2), 247-266.

Godfrey-Smith, P. (2009). Darwinian populations and natural selection. OUP Oxford.

---. (2001). Three kinds of adaptationism. Adaptationism and optimality, 335-357.

Gould, Stephen J. (1977). Ontogeny and Phylogeny. Cambridge, MA: Harvard University Press.

---. (1980a). The Evolutionary Biology of Constraint. Daedalus, 109:39-52.

---. (1980b). The Promise of Paleobiology as a Nomothetic, Evolutionary Discipline.

Paleobiology, 6 (1):96-118. 
---. (1989a). Wonderful Life: The Burgess Shale and the Nature of History. New York, NY: W.W. Norton and Company.

---. (1989b). A Developmental Constraint in Cerion, with comments on the definition and interpretation of constraint in evolution. Evolution, 43:516-39.

---. (2002). The Structure of Evolutionary Theory. Massachusetts, US: Harvard University Press.

Gould, S. J., \& Lewontin, R. C. (1979). The spandrels of San Marco and the Panglossian paradigm: a critique of the adaptationist programme. Proceedings of the Royal Society of London B: Biological Sciences, 205(1161), 581-598.

Gould, S. J., \& Eldredge, N. (1977). Punctuated equilibria: the tempo and mode of evolution reconsidered. Paleobiology, 3(02), 115-151.

Grantham, T. (2007). Is macroevolution more than successive rounds of microevolution?. Palaeontology, 50(1), 75-85.

Griffiths, P. E. (1999). Squaring the circle: natural kinds with historical essences. Ed Wilson, R. A. Species: New interdisciplinary essays.

Hall, B. K. (2012). Parallelism, deep homology, and evo-devo. Evolution \& Development, $14,33-39$.

Hull, D. L. (1976). Are species really individuals?. Systematic Biology, 25(2), 174-191.

Huss, J. (2009). The Shape of Evolution: The MBL Model and Clade Shape. IN: Sepkoski \& Ruse (ed) The Paleobiological Revolution. University of Chicago Press.

Inkpen, R., \& Turner, D. (2012). The topography of historical contingency. Journal of the Philosophy of History, 6(1), 1-19.

Jacob, François (1977). Evolution and Tinkering. Science, 196 (4295): 1161-66.

Langenheder, Silke and Anna J. Székley (2011). Species sorting and neutral processes are both important during the initial assembly of bacterial communities. The International Society for Microbial Ecology Journal, 5:1086-94.

Lewens, T. (2009). Seven types of adaptationism. Biology \& Philosophy, 24(2), 161-182.

Lewontin, R. C. (1970). The units of selection. Annual review of ecology and systematics, 118.

Louis, A. A. (2016). Contingency, convergence and hyper-astronomical numbers in biological evolution. Studies in History and Philosophy of Science Part C: Studies in History and Philosophy of Biological and Biomedical Sciences.

Mäthger, L. M., Barbosa, A., Miner, S., \& Hanlon, R. T. (2006). Color blindness and contrast perception in cuttlefish (Sepia officinalis) determined by a visual sensorimotor assay. Vision research, 46(11), 1746-1753. 
McGhee, George. R. (2011). Convergent evolution: limited forms most beautiful. MIT Press. Millstein, Roberta (2000). Chance and Macroevolution. Philosophy of Science, 67: 603-24.

Morris, S. C. (2003). Life's solution: inevitable humans in a lonely universe. Cambridge University Press.

Muller, H.J. (1922). Variation due to change in the individual gene. The American Naturalist, 56:32-50.

Okasha, Samir (2015). Population Genetics. In The Stanford Encyclopedia of Philosophy, Edward N. Zalta (ed.). URL= <http://plato.stanford.edu/archives/fall2015/entries/populationgenetics/>.

Okasha, S. (2006). Evolution and the levels of selection (Vol. 16). Oxford: Clarendon Press.

Osorio, D., Smith, A. C., Vorobyev, M., \& Buchanan-Smith, H. M. (2004). Detection of fruit and the selection of primate visual pigments for color vision. The American Naturalist, 164(6), 696-708.

Plutynski, A. (2007). Drift: A historical and conceptual overview. Biological Theory, 2(2), 156-167.

Powell, R., \& Mariscal, C. (2014). There is grandeur in this view of life: the biophilosophical implications of convergent evolution. Acta Biotheor., 62, 115-121.

Powell, R., \& Mariscal, C. (2015). Convergent evolution as natural experiment: the tape of life reconsidered. Interface focus, 5(6).

Powell, R. (2009). Contingency and convergence in macroevolution: a reply to John Beatty. The Journal of Philosophy, 106(7), 390-403.

Powell, R. (2007). Is Convergence More than an Analogy? Homoplasy and its Implications for Macroevolutionary Predictability. Biology and Philosophy, 22:565-578.

Regan, B. C., Julliot, C., Simmen, B., Vienot, F., Charles-Dominique, P., \& Mollon, J. D. (2001). Fruits, foliage and the evolution of primate colour vision. Philosophical Transactions of the Royal Society B: Biological Sciences, 356(1407), 229-283.

Roth, Paul A. (2008) "Varieties and Vagaries of Historical Explanation" (PDF) Journal of the Philosophy of History 2. pp. 214-226.

Simpson, G. G. (1944). Tempo and mode in evolution. Columbia University Press.

Smart, J. J. C. (1959). Can biology be an exact science?. Synthese, 11(4), 359-368.

Sterelny, K. (forthcoming). Contingency \& History. Philosophy of Science.

Sterelny, K. (2005). Another view of life. Studies in History and Philosophy of Science Part C: Studies in History and Philosophy of Biological and Biomedical Sciences, 36(3), 585-593. 
Sterelny, K. (2001). Dawkins vs. Gould: Survival of the Fittest. London, UK: Icon Books Ltd.

Travisano, Michael, Judith A. Mongold, Albert F. Bennett, and Richard Lenski (1995).

Experimental tests of the roles of adaptation, chance, and history in evolution. Science, 267 (5194): 87-90.

Turner, Derek (2011a). Gould's replay revisited. In Biol. Philos 26: 65-79.

---. (2011b). Paleontology: A Philosophical Introduction. Cambridge, UK: Cambridge University Press.

---. (2015). Historical contingency and the explanation of evolutionary trends. In C. Malaterre and P. Braillard (eds.), Biological Explanation: An Enquiry into the Diversity of Explanatory Patterns in the Life Sciences, 73-90. London, UK: Springer Dordrecht Heidelberg.

Vlahos, L. M., Knott, B., Valter, K., \& Hemmi, J. M. (2014). Photoreceptor topography and spectral sensitivity in the common brushtail possum (Trichosurus vulpecula). Journal of Comparative Neurology, 522(15), 3423-3436. 